\title{
Article \\ Long-Term Mortality after Histoplasma Infection in People with HIV
}

\author{
Joseph Cherabie $\mathbb{( D )}$, Patrick Mazi, Adriana M. Rauseo $(\mathbb{D}$, Chapelle Ayres, Lindsey Larson, Sasinuch Rutjanawech, \\ Jane O’Halloran, Rachel Presti, William G. Powderly (1) and Andrej Spec*
}

Citation: Cherabie, J.; Mazi, P.; Rauseo, A.M.; Ayres, C.; Larson, L.; Rutjanawech, S.; O'Halloran, J.; Presti, R.; Powderly, W.G.; Spec, A. Long-Term Mortality after Histoplasma Infection in People with HIV. J. Fungi 2021, 7, 369. https:// doi.org/10.3390/jof7050369

Academic Editor: George Deepe

Received: 9 April 2021

Accepted: 6 May 2021

Published: 8 May 2021

Publisher's Note: MDPI stays neutral with regard to jurisdictional claims in published maps and institutional affiliations.

Copyright: (c) 2021 by the authors. Licensee MDPI, Basel, Switzerland. This article is an open access article distributed under the terms and conditions of the Creative Commons Attribution (CC BY) license (https:/ / creativecommons.org/licenses/by/ $4.0 /)$.
Division of Infectious Diseases, Department of Medicine, Washington University School of Medicine, St. Louis, MO 63110, USA; jcherabie@wustl.edu (J.C.); pmazi@wustl.edu (P.M.); a.rauseoacevedo@wustl.edu (A.M.R.); ayreschapelle@wustl.edu (C.A.); lindseylarson@wustl.edu (L.L.); sasinuchr@wustl.edu (S.R.); janeaohalloran@wustl.edu (J.O.); prestir@wustl.edu (R.P.); wpowderly@wustl.edu (W.G.P.)

* Correspondence: andrejspec@wustl.edu; Tel.: +1-314-747-1725

\begin{abstract}
Histoplasmosis is a common opportunistic infection in people with HIV (PWH); however, no study has looked at factors associated with the long-term mortality of histoplasmosis in PWH. We conducted a single-center retrospective study on the long-term mortality of PWH diagnosed with histoplasmosis between 2002 and 2017. Patients were categorized into three groups based on length of survival after diagnosis: early mortality (death $<90$ days), late mortality (death $\geq 90$ days), and long-term survivors. Patients diagnosed during or after 2008 were considered part of the modern antiretroviral therapy (ART) era. Insurance type (private vs. public) was a surrogate indicator of socioeconomic status. Out of 54 PWH infected with histoplasmosis, overall mortality was 37\%; 14.8\% early mortality and $22.2 \%$ late mortality. There was no statistically significant difference in survival based on the availability of modern ART $(p=0.60)$. Insurance status reached statistical significance with $38 \%$ of survivors having private insurance versus only $8 \%$ having private insurance in the late mortality group $(p=0.05)$. High mortality persists despite the advent of modern ART, implicating a contribution from social determinants of health, such as private insurance. Larger studies are needed to elucidate the role of these factors in the mortality of PWH.
\end{abstract}

Keywords: HIV; Histoplasma; antiretroviral therapy; mortality

\section{Introduction}

Histoplasmosis is the most prevalent endemic mycosis in the United States; an estimated 50,778 histoplasmosis-associated hospitalizations occurred between 2001 and 2012 with a trend towards increased incidence [1]. The incidence of histoplasmosis among adults in the US is highest in the Midwest with 5.1 cases per 100,000 population and common worldwide among people with HIV / AIDS (PWH) without access to anti-retroviral therapy (ART) [2].

Histoplasmosis carries a high 90 -day mortality of $16 \%$, with mortality increasing among immunocompromised individuals up to $24 \%$ [3]. Previous studies have found that histoplasmosis, specifically disseminated histoplasmosis, carries a high mortality among PWH greater than $30 \%$, although many of these studies were performed prior to the era of modern ART $[4,5]$. In other opportunistic mycoses, late mortality appears to be significantly higher than the early mortality initially observed [6].

While multiple studies have assessed factors associated with early mortality from histoplasmosis among PWH [7-10], to the best of our knowledge no studies have thus far looked at factors associated with long-term mortality, particularly within the era of modern ART. 


\section{Materials and Methods}

This is a retrospective cohort study of patients diagnosed with Histoplasma infection at Barnes-Jewish Hospital (BJH) in St. Louis, Missouri. BJH is a 1368-bed tertiary care academic hospital. While located in an urban setting, BJH has a large suburban and rural catchment area. This study was approved by the Washington University School of Medicine Human Research Protection Office, with a waiver of informed consent.

The cohort consisted of all individuals diagnosed with Histoplasma capsulatum infection aged 18 or older within BJH from 1 January 2002 until 31 December 2017. Histoplasma infection required one of the following: (1) positive Histoplasma antigen from urine, serum, CSF, or other body fluid; (2) microbiologic isolation of $H$. capsulatum from any source; (3) coding for International Classification of Diseases (ICD) 9th (115.x) or 10th (B39.X) codes for Histoplasma infection; or (4) positive Histoplasma antibody. Cases were confirmed independently by two study investigators to ensure cases met criteria for proven or probable histoplasmosis by MSG/EORTC criteria [11]. Exclusion criteria consisted of the following: (1) if antigen or antibody testing was positive in the setting of another fungal infection confirmed by culture or histopathology; (2) if an antigen was positive at a low or indeterminate level and the case was determined to be a false positive by the treating infectious diseases team at the time and/or by the two study investigators during independent review; (3) if there was a diagnosis of presumed ocular histoplasmosis syndrome without other evidence of Histoplasma infection; and (4) if the patient presented with fibrosing mediastinitis. $\mathrm{PWH}$ were then sub-selected if they had a positive 3rd or 4th generation HIV screening test with positive confirmatory HIV viral load test. Additional information pertaining to each patient's HIV infection was obtained from the patients' charts, including CD4 count and HIV viral load at time of Histoplasma infection diagnosis, HIV viral load at the time of last encounter, anti-retroviral experience prior to diagnosis, antiretroviral prescribed at the time of diagnosis, insurance status, history of other opportunistic infections (OI), history of substance use, and history of psychiatric illness. Data were collected through automated extraction from the electronic medical record and manual chart review. Other participant data collected included patient demographics, clinical presentation, organ system involvement in infection, and mortality.

The primary outcome for our study was all-cause late mortality, defined as deaths occurring at or after 90 days of histoplasmosis diagnosis. The secondary outcome was all-cause early mortality, defined as deaths occurring within 90 days of histoplasmosis diagnosis. Date of death was obtained from the electronic medical record and Social Security Death Index. The Social Security Death Index includes deaths through 2014 with deaths after 2014 obtained through the electronic medical record system. If there was no date of death on record, then it was assumed the patient survived until the last day the patient interacted with the healthcare system and was right-censored after that date. Pre-modern ART was defined as a participant having been diagnosed with histoplasmosis before 2008, and modern ART was a participant having been diagnosed from 2008 onwards, with 2008 marking the advent of darunavir and integrase inhibitors as standard ART. A participant was considered virally suppressed if they had a HIV viral load $<50$ copies $/ \mathrm{mL}$. Insurance status was categorized into two groups: those with private insurance and individuals with government insurance (Medicare or Medicaid) or no insurance.

Categorical variables were analyzed using Pearson Chi squared analysis. Continuous variables were analyzed using Mann-Whitney U non-parametric testing to account for small numbers and lack of normal distribution. $p$-values $\leq 0.05$ were considered statistically significant. All statistical analysis was performed using SPSS V25 (IBM, Armonk, NY, USA).

\section{Results}

Fifty-four individuals were found to have both histoplasmosis as well as HIV infection within our study period; $68.5 \%$ were male and $59.3 \%$ were Black. There was no statistically significant difference between survival groups with respect to age $(p=0.28)$, gender $(p=0.91)$ or race $(p=0.11)$ (Table 1$)$. 
Table 1. Baseline characteristics and presentation of PWH with histoplasmosis $(n=54)$ among three groups (survivors, early mortality, and late mortality) 2002-2017.

\begin{tabular}{|c|c|c|c|c|}
\hline & $\begin{array}{c}\text { Survived } \\
n=34(63.0 \%)\end{array}$ & $\begin{array}{l}\text { Early Mortality } \\
\qquad n=8(14.8 \%)\end{array}$ & $\begin{array}{l}\text { Late Mortality } \\
n=12(22.2 \%)\end{array}$ & $p$-Value \\
\hline Male & $23(67 \%)$ & $6(75 \%)$ & $8(67 \%)$ & 0.91 \\
\hline Age (years, median, IQR) & $43(32,51)$ & $41(36,49)$ & $36(25,40)$ & 0.28 \\
\hline \multicolumn{4}{|l|}{ 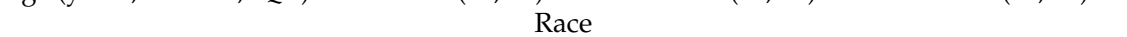 } & 0.11 \\
\hline Black & $16(47 \%)$ & $7(87 \%)$ & $9(75 \%)$ & \\
\hline Non-Black & $17(50 \%)$ & $1(13 \%)$ & $2(17 \%)$ & \\
\hline \multicolumn{5}{|c|}{ Site of infection } \\
\hline CNS & $3(9 \%)$ & $0(0 \%)$ & $0(0 \%)$ & 0.39 \\
\hline Pulmonary & $24(71 \%)$ & $6(75 \%)$ & $7(58 \%)$ & 0.67 \\
\hline Bloodstream & $15(44 \%)$ & $3(38 \%)$ & $8(67 \%)$ & 0.33 \\
\hline Disseminated disease & $26(76 \%)$ & $5(63 \%)$ & $11(92 \%)$ & 0.29 \\
\hline \multicolumn{5}{|c|}{ Presenting symptoms } \\
\hline Fever & $26(76 \%)$ & $4(50 \%)$ & $10(83 \%)$ & 0.22 \\
\hline Cough & $17(50 \%)$ & $4(50 \%)$ & $5(42 \%)$ & 0.88 \\
\hline Night sweats & $10(29 \%)$ & $1(13 \%)$ & $5(42 \%)$ & 0.38 \\
\hline Dyspnea & $13(38 \%)$ & $3(38 \%)$ & $6(50 \%)$ & 0.76 \\
\hline Chest pain & $6(18 \%)$ & $0(0 \%)$ & $3(25 \%)$ & 0.33 \\
\hline Arthralgias & $3(9 \%)$ & $0(0 \%)$ & $1(8 \%)$ & 0.69 \\
\hline Dysphagia & $5(15 \%)$ & $2(25 \%)$ & $2(17 \%)$ & 0.78 \\
\hline Weight loss & $22(65 \%)$ & $5(63 \%)$ & $5(42 \%)$ & 0.37 \\
\hline GI symptoms & $23(68 \%)$ & $4(50 \%)$ & $8(67 \%)$ & 0.64 \\
\hline
\end{tabular}

Overall mortality was 37\%. A total of $14.8 \%(n=8)$ of individuals expired within 90 days of histoplasmosis diagnosis with a median length of survival of 13.5 days (IQR $2.5-41)$, while $22.2 \%(n=12)$ expired on or after 90 days with a median length of survival of 302 days (IQR 153-710.5). Death occurred within 30 days in $9.3 \%$ of individuals $(n=5)$, in 31-90 days in $5.5 \%$ of individuals $(n=3)$, in $91-365$ days in $13 \%$ of individuals $(n=7)$, and greater than 365 days in $9.3 \%$ of individuals $(n=5)$. Death occurring within 1 year had a median survival of 86 days (IQR 6-177) and after 1 year had a median survival of 798 days (IQR 418-993).

Private insurance was a factor more common to the survival group at $38 \%$ compared to $8 \%$ of the late mortality group that had private insurance $(p=0.05)$ (Table 2$)$. There was no significant difference between survivors and late mortality individuals with respect to history of substance use (survivors $n=13,38 \%$ survivors vs. late mortality individuals $n=2,17 \%, p=0.17$ ) or history of psychiatric illness (survivors $n=4,12 \%$, vs. late mortality individuals $n=0,0 \%, p=0.21$ ).

Table 2. Baseline HIV-related characteristics of PWH with histoplasmosis $(n=46)$ among two groups (survivors and late mortality) 2002-2017.

\begin{tabular}{|c|c|c|c|}
\hline & Survived $(n=34)$ & Late Mortality $(n=12)$ & $p$-Value \\
\hline Median CD4 count (cells $/ \mathrm{mm}^{3}$, median, IQR) & $12(6,46)$ & $26(8,52)$ & 0.52 \\
\hline Median HIV viral load at diagnosis (copies/mL, median, IQR) & $5.5(4.4,6.2)$ & $5.2(3.8,5.5)$ & 0.25 \\
\hline Median HIV viral load at last observation (copies/mL, median, IQR) & $2.0(0,4.5)$ & $4.1(2.6,5.5)$ & 0.01 \\
\hline New HIV diagnosis at diagnosis & $18(53 \%)$ & $3(25 \%)$ & 0.10 \\
\hline Median time between HIV and Histoplasma diagnoses (years, median, IQR) & $0.2(0,13.6)$ & $3.5(0.1,5.3)$ & 0.87 \\
\hline ART-experienced & $14(41 \%)$ & $7(58 \%)$ & 0.19 \\
\hline Modern ART & $12(35 \%)$ & $4(33 \%)$ & 0.90 \\
\hline HIV viral load suppressed at last observation & $14(41 \%)$ & $2(17 \%)$ & 0.13 \\
\hline HIV viral load suppressed at diagnosis & $1(3 \%)$ & $2(17 \%)$ & 0.10 \\
\hline History of previous OIs & $17(50 \%)$ & $9(75 \%)$ & 0.13 \\
\hline Pneumocystis pneumonia & $10(29 \%)$ & $2(17 \%)$ & \\
\hline Oral Candidiasis & $4(12 \%)$ & $2(17 \%)$ & \\
\hline CMV & $2(6 \%)$ & $2(17 \%)$ & \\
\hline Private insurance & $13(38 \%)$ & $1(8 \%)$ & 0.05 \\
\hline History of substance use & $13(38 \%)$ & $2(17 \%)$ & 0.17 \\
\hline History of psychiatric illness & $4(12 \%)$ & $0(0 \%)$ & 0.21 \\
\hline
\end{tabular}




\subsection{Disease Presentation}

Disseminated histoplasmosis was the most common disease presentation within all groups, occurring in $76 \%$ of survivors $(n=26), 63 \%$ of individuals with early mortality $(n=5)$, and $92 \%$ of individuals with late mortality $(n=11)$, with no statistically significant difference between the three groups $(p=0.29)$ (Table 1). Localized pulmonary infection was common within all three groups $(n=37,68.5 \%, p=0.67)$, and central nervous system (CNS) infection was seen in three patients, all of whom survived. As for presenting symptoms, fever was the most common $(74.1 \%)$, followed by gastrointestinal symptoms $(64.8 \%)$ and cough $(48.1 \%)$.

\subsection{HIV Characteristics}

While the median time in years from HIV diagnosis to histoplasmosis diagnosis in survivors was 3.3 years shorter than among late mortality individuals $(0.2$ years, IQR $0-13.6$ years vs. 3.5 years, IQR $0.1-5.3$ years), this difference was not statistically significant $(p=0.87)$ (Table 2). Having a new HIV diagnosis at the time of Histoplasma diagnosis was more common among survivors $(n=18,53 \%)$ compared to late-mortality individuals $(n=3$, $25 \%)$, but this also did not meet statistical significance $(p=0.10)$.

HIV viral load at the time of diagnosis with histoplasmosis did not vary significantly between survivors (5.5 median log copies/mL, IQR 4.4-6.2) and individuals with late mortality (5.2 median log copies/mL, IQR 3.8-5.5) $(p=0.25)$. Similarly, there was no statistically significant difference in viral load suppression (survivors $n=1,3 \%$ vs. late mortality $n=2,17 \%)(p=0.10)$ or median CD4 count at the time of Histoplasma infection diagnosis (median CD4 12 cells $/ \mathrm{mm}^{3}$, IQR 6-46 in survivors vs. median CD4 26 cells $/ \mathrm{mm}^{3}$, IQR 8-52 in late mortality individuals, $p=0.52$ ).

Median HIV viral load at last observation was significantly lower in survivors (2 log copies / $\mathrm{mL}$, IQR 0-4.5) compared to the late mortality individuals (4.1 log copies $/ \mathrm{mL}$, IQR 2.6-5.5) $(p=0.01)$. Survivors $(n=14,41 \%)$ were more than twice as likely to have HIV viral load suppression at the time of last observation compared to individuals with late mortality $(n=2,17 \%)$; however, this difference was not statistically significant $(p=0.13)$.

\subsection{ART Experience and Opportunistic Infections}

ART experience made no difference with respect to survival ( $n=14,41 \%$ of survival group vs. $n=7,58 \%$ of late mortality group, $p=0.19)$, nor did being diagnosed with Histoplasma infection in the era of modern ART ( $n=12,35 \%$, of survival group vs. $n=4$, $33 \%$ of late mortality group, $p=0.90$ ). Previous opportunistic infections (OIs) occurred in $50 \%$ of the survivor group $(n=17)$ vs. $75 \%$ of the late mortality group $(n=9)$, with no significant difference in OI occurrence between the two groups $(p=0.13)$. The most common other opportunistic infections overall were Pneumocystis pneumonia, followed by oral candidiasis and Cytomegalovirus infection. Of all the individuals with late mortality and OIs, three had no death certificates to indicate cause of death, while three had one or more OIs other than histoplasmosis active at the time of death.

\section{Discussion}

This is the first study assessing the long-term mortality of histoplasmosis in a US population of PWH. Despite the resource-rich environment of US healthcare and most of our study occurring during the modern era of ART, overall mortality was $37 \%$. This is a similar mortality rate to the resource-limited environments of French Guiana, Guatemala, and Brazil-all-cause mortality of $41 \%, 43.6 \%$, and $30.2 \%$, respectively [11-13]. In the French Guiana and Guatemalan cohorts, approximately $75 \%$ of deaths occurred within a year of histoplasmosis diagnosis. Our study showed similar findings to these studies, suggesting that the first 12 months is a critical period for $\mathrm{PWH}$.

The specific cause of each patient's death is uncertain due to many OIs being listed as active at the time of death with causes of death being multifactorial. This is often the case in patients with uncontrolled HIV. Despite this, we identified that HIV viral load at the 
time of diagnosis with histoplasmosis did not vary significantly between survivors and individuals with late mortality. Similarly, there was no statistically significant difference in viral load suppression or median CD4 count at the time of Histoplasma infection diagnosis. Patients presenting in the French Guiana and Guatemala cohorts also had uncontrolled HIV with median CD4 counts of 31 and 25 , respectively.

The advent of integrase inhibitors in 2007-2008 ushered in the modern era of ART. Modern ART is better tolerated, leads to more rapid viral suppression, and has higher uptake than historical formulations [14,15]. While the advent of modern ART has had an effect on the mortality of PWH with other mycoses such as Cryptococcus [6], in our cohort, being diagnosed with histoplasmosis in the era of modern ART had no effect on survival.

To our knowledge, our study is the first to look at social factors such as private insurance, mental health, and substance use on the mortality of PWH with histoplasmosis. A statistically significant improvement in mortality was observed in patients having private insurance vs. government or no insurance. Health insurance itself is a reflection on socio-economic status, especially within the US, where individuals with insurance report significantly greater access and quality of health services compared to individuals who are uninsured, especially with respect to accessing specialist appointments when private insurance was compared to Medicaid and uninsured individuals [16]. Negative outcomes are common among PWH who are uninsured, especially with regard to limited access to indicated ART regimens, case management, and mental health services [17,18].

Delayed mortality from OIs in PWH is not unique to just histoplasmosis. In PWH diagnosed with Cryptococcus infection, $66 \%$ of deaths occurred after 90 days [6]. Similar to our study, survivors were more likely to have private insurance. In contrast to our study, the authors found improved mortality associated with viral suppression at last follow up. Additionally, they found an improvement in outcomes in the modern ART era and higher rates of substance use and mental illness in non-survivors [6]. The opposite was seen in our study, with survivors having higher rates of substance use and a history of psychiatric illness, and although neither finding was statistically significant, these findings suggest these factors may not affect mortality in histoplasmosis patients in the same way as cryptococcosis.

Other OIs in the era of modern ART do not show similar delayed mortality as observed in histoplasmosis and cryptococcosis. A study of 1264 Taiwanese PWH in the era of modern ART found $21 \%$ of patients had OIs, with the most common being P. jirovecii (43.4\%), followed by Cytomegalovirus (10.4\%), tuberculosis ( $8.1 \%)$, and candidiasis (6.9\%). The majority of OI events $(91.7 \%)$ developed within 90 days and all-cause mortality was $4.4 \% ; 83.9 \%$ being OI related etiologies with no significant difference between mortality rate at 90 days, between 91 and 180 days, and $>180$ days [19]. These data contrast with our cohort of PWH with histoplasmosis as their diagnosed OI, where the majority of mortality occurred more than 90 days after diagnosis.

Small sample size limited our ability to perform multivariate analyses among all three groups. The early mortality group was too small, with wide confidence intervals in any comparison, limiting comparisons to the survivor and the late mortality groups. Another limitation is that our data did not capture whether a patient's insurance status changed after their hospitalization. Insurance status is subject to change over time as the result of many factors; however, due to the size of our study, we considered this a definitive variable. This study was performed at a single, academic medical center located in the Midwest, which may limit the generalizability of results to other centers with variable endemicity of histoplasmosis and to regions where different OIs are more endemic. Lastly, and possibly most importantly, we were limited by our lack of definitive cause of death due to the retrospective nature of the study.

In conclusion, the mortality of PWH diagnosed with histoplasmosis remains high, even in the resource-rich environment of the US medical system, with comparable gross mortality to similar cohorts in more resource-limited settings $[12,13,20]$. Despite access to accurate diagnostics, modern ART, and effective antifungal treatment regimens, our 
US cohort did not trend towards improved mortality as we transitioned in the era of modern ART, unlike the cohorts from French Guiana, Guatemala, and Brazil. Patients with government or no insurance were five times more likely to have a late death compared to patients with private insurance. Though these are complex medical situations with many confounders, we hypothesize private insurance is a surrogate for a patient's ability to access and utilize the US medical system. That PWH in our cohort became immunocompromised enough to be diagnosed with histoplasmosis and then to experience delayed mortality beyond the duration of histoplasmosis treatment is likely a failure of the US medical system to maximize the care this population needs. We recommend additional research including broadening our cohort to other resource-rich settings to further test our hypothesis and more fully elucidate the deficiencies in our HIV care model.

Author Contributions: Conceptualization, J.C., J.O., R.P. and A.S.; Data curation, L.L.; Formal analysis, C.A. and L.L.; Funding acquisition, W.G.P.; Investigation, J.C.; Project administration, C.A. and L.L.; Supervision, A.S.; Writing-original draft, J.C., P.M., A.M.R., L.L., S.R. and A.S.; Writingreview and editing, J.C., P.M., A.M.R., C.A., J.O., R.P., W.G.P. and A.S. All authors have read and agreed to the published version of the manuscript.

Funding: Research reported in this publication was supported by the Washington University Institute of Clinical and Translational Sciences grant UL1TR002345 from the National Center for Advancing Translational Sciences (NCATS) of the National Institutes of Health (NIH). The content is solely the responsibility of the authors and does not necessarily represent the official view of the NIH.

Institutional Review Board Statement: This study was approved by the Washington University School of Medicine Human Research Protection Office, with a waiver of informed consent.

Conflicts of Interest: Powderly reports grant support from Merck Labs and consulting work with Merck Labs and Gilead. Spec reports grant support with Astellas, consulting work with Viamet, Mayne, and Scynexis. All other authors declare no conflict of interest.

\section{References}

1. Benedict, K.; Derado, G.; Mody, R.K. Histoplasmosis-Associated Hospitalizations in the United States, 2001-2012. Open Forum Infect. Dis. 2016, 3, ofv219. [CrossRef] [PubMed]

2. Baddley, J.W.; Winthrop, K.L.; Patkar, N.M.; Delzell, E.; Beukelman, T.; Xie, F.; Chen, L.; Curtis, J.R. Geographic distribution of endemic fungal infections among older persons, United States. Emerg. Infect. Dis. 2011, 17, 1664-1669. [CrossRef] [PubMed]

3. Franklin, A.D.; Larson, L.; Rauseo, A.M.; Rutjanawech, S.; Hendrix, M.J.; Powderly, W.J.; Spec, A. A comparison of presentations and outcomes of histoplasmosis across patients with varying immune status. Med. Mycol. 2021. [CrossRef] [PubMed]

4. Couppie, P.; Aznar, C.; Carme, B.; Nacher, M. American histoplasmosis in developing countries with a special focus on patients with HIV: Diagnosis, treatment, and prognosis. Curr. Opin. Infect. Dis. 2006, 19, 443-449. [CrossRef] [PubMed]

5. Huber, F.; Nacher, M.; Aznar, C.; Pierre-Demar, M.; El Guedj, M.; Vaz, T.; Vantilcke, V.; Mahamat, A.; Magnien, C.; Chauvet, E.; et al. AIDS-related Histoplasma capsulatum var. capsulatum infection: 25 years experience of French Guiana. AIDS 2008, 22, 1047-1053. [CrossRef] [PubMed]

6. Hevey, M.A.; Presti, R.M.; O’Halloran, J.A.; Larson, L.; Raval, K.; Powderly, W.G.; Spec, A. Mortality After Cryptococcal Infection in the Modern Antiretroviral Therapy Era. J. Acquir. Immune. Defic. Syndr. 2019, 82, 81-87. [CrossRef] [PubMed]

7. Couppie, P.; Sobesky, M.; Aznar, C.; Bichat, S.; Clyti, E.; Bissuel, F.; El Guedj, M.; Alvarez, F.; Demar, M.; Louvel, D.; et al. Histoplasmosis and Acquired Immunodeficiency Syndrome: A Study of Prognostic Factors. Clin. Infect. Dis. 2004, 38, 134-138. [CrossRef] [PubMed]

8. Boigues, B.C.S.; Paniago, A.M.M.; Lima, G.M.E.; Nunes, M.O.; Uehara, S.N.O. Clinical outcomes and risk factors for death from disseminated histoplasmosis in patients with AIDS who visited a high-complexity hospital in Campo Grande, MS, Brazil. Rev. Soc. Bras. Med. Trop. 2018, 51, 155-161. [CrossRef] [PubMed]

9. Baddley, J.W.; Sankara, I.R.; Rodriquez, J.M.; Pappas, P.G.; Many, W.J., Jr. Histoplasmosis in HIV-infected patients in a southern regional medical center: Poor prognosis in the era of highly active antiretroviral therapy. Diagn. Microbiol. Infect. Dis. 2008, 62, 151-156. [CrossRef] [PubMed]

10. Adenis, A.; Nacher, M.; Hanf, M.; Vantilcke, V.; Boukhari, R.; Blachet, D.; Demar, M.; Aznar, C.; Carme, B.; Couppie, P. HIVassociated histoplasmosis early mortality and incidence trends: From neglect to priority. PLoS Negl. Trop. Dis. 2014, 8, e3100. [CrossRef] [PubMed]

11. Samayoa, B.; Roy, M.; Cleveland, A.A.; Medina, N.; Lau-Bonilla, D.; Scheel, C.M.; Gomez, B.L.; Chiller, T.; Arathoon, E. High Mortality and Coinfection in a Prospective Cohort of Human Immunodeficiency Virus/Acquired Immune Deficiency Syndrome Patients with Histoplasmosis in Guatemala. Am. J. Trop. Med. Hyg. 2017, 97, 42-48. [CrossRef] [PubMed] 
12. Nacher, M.; Valdes, A.; Adenis, A.; Blaizot, R.; Abboud, P.; Demar, M.; Djossou, F.; Epelboin, L.; Misslin, C.; Ntab, B.; et al. Disseminated Histoplasmosis in HIV-Infected Patients: A Description of 34 Years of Clinical and Therapeutic Practice. J. Fungi. (Basel) 2020, 6, 164. [CrossRef] [PubMed]

13. Damasceno, L.S.; Ramos, A.N., Jr.; Alencar, C.H.; Gonçalves, M.V.; de Mesquita, J.R.; Soares, A.T.; Coutinho, A.G.; Dantas, C.C.; Leitão, T.d.M.J.S. Disseminated histoplasmosis in HIV-infected patients: Determinants of relapse and mortality in a north-eastern area of Brazil. Mycoses 2014, 57, 406-413. [CrossRef] [PubMed]

14. Ghosh, A.; Osswald, H.; Prato, G. Recent Progress in the Development of HIV-1 Protease Inhibitors for the Treatment of HIV/AIDS. J. Med. Chem. 2016, 59, 5172-5208. [CrossRef] [PubMed]

15. Psichogiou, M.; Poulakou, G.; Basoulis, D.; Paraskevis, D.; Markogiannakis, A.; Daikos, G.L. Recent Advances in Antiretroviral Agents: Potent Integrase Inhibitors. Curr. Pharm. Des. 2017, 23, 2552-2567. [CrossRef] [PubMed]

16. Nguyen, K.H.; Sommers, B.D. Access and Quality of Care by Insurance Type for Low-Income Adults Before the Affordable Care Act. Am. J. Public Health 2016, 106, 1409-1415. [CrossRef] [PubMed]

17. Weiser, J.; Beer, L.; Frazier, E.L.; Patel, R.; Dempsey, A.; Hauck, H.; Skarbinski, J. Service Delivery and Patient Outcomes in Ryan White HIV / AIDS Program-Funded and -Nonfunded Health Care Facilities in the United States. JAMA Intern. Med. 2015, 175, 1650-1659. [CrossRef] [PubMed]

18. Weiser, J.; Brooks, J.T.; Skarbinski, J.; West, B.T.; Duke, C.C.; Gremel, G.W.; Beer, L. Barriers to Universal Prescribing of Antiretroviral Therapy by HIV Care Providers in the United States, 2013-2014. J. Acquir. Immune. Defic. Syndr. 2017, 74, 479-487. [CrossRef] [PubMed]

19. Lee, C.Y.; Tseng, Y.T.; Lin, W.R.; Chen, Y.H.; Tsai, J.J.; Wang, W.H.; Lu, P.L.; Tsai, H.C. AIDS-related opportunistic illnesses and early initiation of HIV care remain critical in the contemporary HAART era: A retrospective cohort study in Taiwan. BMC Infect. Dis. 2018, 18, 352. [CrossRef] [PubMed]

20. Wheat, L.J.; Freifeld, A.G.; Kleiman, M.B.; Baddley, J.W.; McKinsey, D.S.; Loyd, J.E.; Kauffman, C.A. Infectious Diseases Society of America. Clinical practice guidelines for the management of patients with histoplasmosis: 2007 update by the Infectious Diseases Society of America. Clin. Infect. Dis. 2007, 45, 807-825. [CrossRef] [PubMed] 